\title{
GFF3sort: a novel tool to sort GFF3 files for tabix indexing
}

\author{
Tao Zhu(D, Chengzhen Liang, Zhigang Meng, Sandui Guo* and Rui Zhang*
}

\begin{abstract}
Background: The traditional method of visualizing gene annotation data in JBrowse is converting GFF3 files to JSON format, which is time-consuming. The latest version of JBrowse supports rendering sorted GFF3 files indexed by tabix, a novel strategy that is more convenient than the original conversion process. However, current tools available for GFF3 file sorting have some limitations and their sorting results would lead to erroneous rendering in JBrowse.

Results: We developed GFF3sort, a script to sort GFF3 files for tabix indexing. Specifically designed for JBrowse rendering, GFF3sort can properly deal with the order of features that have the same chromosome and start position, either by remembering their original orders or by conducting parent-child topology sorting. Based on our test datasets from seven species, GFF3sort produced accurate sorting results with acceptable efficiency compared with currently available tools.

Conclusions: GFF3sort is a novel tool to sort GFF3 files for tabix indexing. We anticipate that GFF3sort will be useful to help with genome annotation data processing and visualization.
\end{abstract}

Keywords: GFF3, JBrowse, Visualization, Tabix

\section{Background}

As a powerful genome browser based on HTML5 and JavaScript, JBrowse has been widely used since released in $2009[1,2]$. According to its configuration document [3], it works by first converting genome annotation data in GFF3 file formats to JSON files by a built-in script "flatfile-to-json.pl", and then rendering visualized element models such as genes, transcripts, repeat elements, etc. The main problem, however, is that this step is extremely time-consuming. The time is proportional to the number of feature elements in GFF3 files (Additional file 1). Even for small genomes like yeast (Saccharomyces cerevisiae), it takes $\sim 10 \mathrm{~s}$ to finish the conversion. For large and deeply annotated genomes such as that of humans, the time increases to more than 15 min. In addition, through the conversion process, a single GFF3 file is converted to thousands of piecemeal JSON files, thus putting a heavy burden on the ability to back up and store data.

\footnotetext{
*Correspondence: guosandui@caas.cn; zhangrui@caas.cn Biotechnology Research Institute, Chinese Academy of Agricultural Sciences, Beijing 100081, China
}

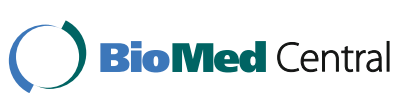

(c) The Author(s). 2017 Open Access This article is distributed under the terms of the Creative Commons Attribution 4.0 International License (http://creativecommons.org/licenses/by/4.0/), which permits unrestricted use, distribution, and reproduction in any medium, provided you give appropriate credit to the original author(s) and the source, provide a link to the Creative Commons license, and indicate if changes were made. The Creative Commons Public Domain Dedication waiver (http://creativecommons.org/publicdomain/zero/1.0/) applies to the data made available in this article, unless otherwise stated. port for indexed GFF3 files has been added [4]. In this strategy, the GFF3 file is compressed with bgzip and indexed with tabix [5], which generates only two data files: a compressed file (.gz) and an index file (.tbi or.csi). Compared with the traditional processing protocol, the whole compression and index process could be finished within a few seconds even for large datasets such as the human genome annotation data (Additional file 1). The tabix tool requires GFF3 files to be sorted by chromosomes and start positions, which could be performed in the GNU sort program or the GenomeTools [6] package (see [7]). When dealing with feature lines in the same chromosome and start position, both of these tools may break ties or return a sort order where child features are placed ahead of their parent feature (Fig. 1a). Although this is still valid for tabix indexing, it would causing erroneous rendering in JBrowse [8] (Fig. 1a). Currently there is no additional options or arguments for current tools to break such tied features by parent-child relationship. In the absence of a suitable bug fix to JBrowse, an alternative sorting tool is needed to resolve this problem. 
a

\begin{tabular}{|c|c|c|}
\hline $\begin{array}{l}1 \text { araport11 exon } \\
1 \text { araport11 five_prime_UTR } \\
1 \text { araport11 } \\
1 \text { arapene } \\
1 \text { araport11 } 11 \text { exNA } \\
1 \text { araport11 } \\
1 \text { five_prime_UTR } \\
1 \text { araport11 } \text { mRNA } \\
1 \text { araport11 CDS } \\
1 \text { araport11 } 11 \text { exs } \\
1 \text { araport11 CDS } \\
1 \text { araport11 exon } \\
1 \text { araport11 exon } \\
1 \text { araport11 five_prime_UTR } \\
1 \text { araport11 CDS } \\
1 \text { araport11 CDS } \\
1 \text { araport11 CDS } \\
1 \text { araport11 exon } \\
1 \text { araport11 exon }\end{array}$ & $\begin{array}{ll}51953 & 52346 \\
51953 & 52238 \\
51953 & 54737 \\
51953 & 54737 \\
52061 & 52730 \\
52061 & 52730 \\
52061 & 54689 \\
52239 & 52346 \\
52434 & 52730 \\
52434 & 52730 \\
52938 & 53183 \\
52938 & 53183 \\
52938 & 53183 \\
52938 & 53021 \\
53022 & 53183 \\
53484 & 53624 \\
53484 & 53624 \\
53484 & 53624 \\
53484 & 53624\end{array}$ & 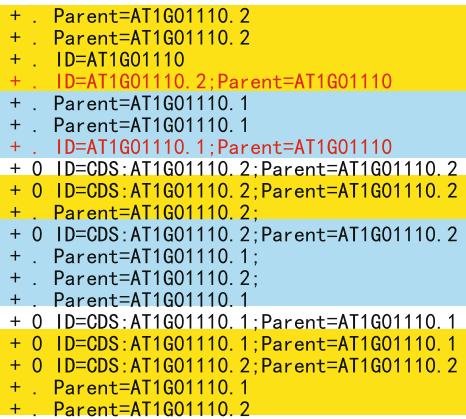 \\
\hline
\end{tabular}

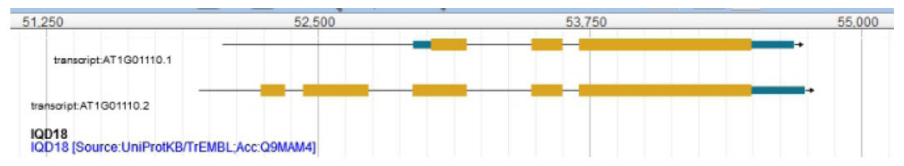

b

Hash Table

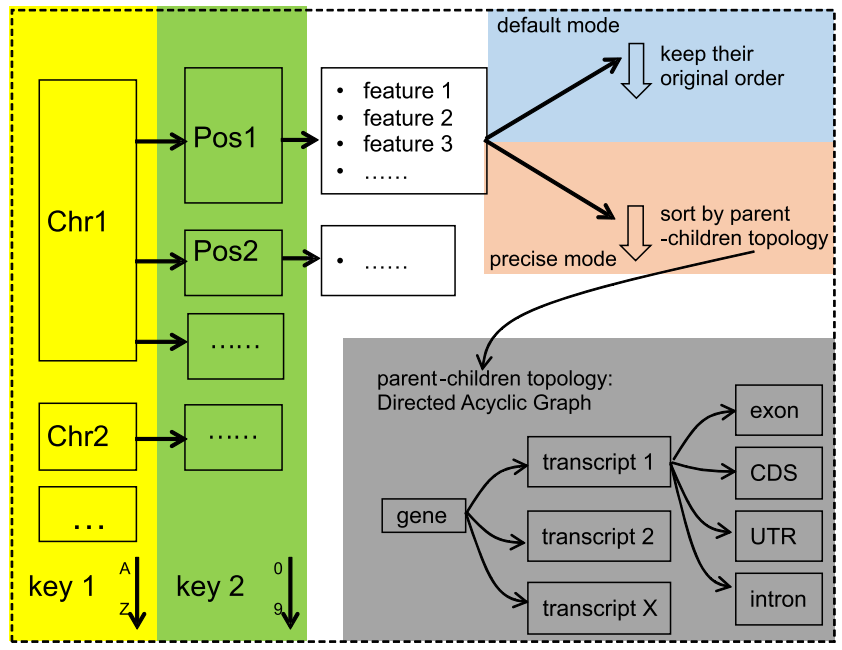
C 1 araport11 gene $\quad 5195354737 .+$. ID=AT1G01110
1 araport11 mRNA $5195354737 .+$. ID=AT1G01110. 2; Parent=AT1 G01110
1 araport11 five_prime_UTR 5195352238 . + . Parent=AT1G01110. 2
1 araport11 exon $5195352346 .+$. Parent=AT1G01110. 2
1 araport11 mRNA $5206154689 .+$. ID=AT1G01110. 1; Parent=AT1G01110
1 araport11 exon $5206152730 .+$. Parent=AT1G01110. 1
1 araport11 five_prime_UTR $5206152730 .+$. Parent=AT1G01110.1
1 araport11 CDS 5223952346 . + 0 ID=CDS:AT1G01110. 2; Parent=AT1G01110. 2
1 araport11 exon $5243452730 .+$. Parent=AT1G01110. 2 .
1 araport11 CDS $\quad 5243452730 .+0$ ID=CDS:AT1G01110. 2 ; Parent=AT1G01110. 2
1 araport11 exon $5293853183 .+$. Parent=AT1G01110. 2 .
1 araport11 CDS $\quad 5293853183^{\circ}+0$ ID $=$ CDS:AT1G01110. 2 ; Parent=AT1G01110. 2
1 araport11 five_prime_UTR $5293853021 .+$ + Parent=AT1G01110. 1
1 araport11 exon 5293853183 . + . Parent=AT1G01110.1.
1 araport11 CDS $\quad 5302253183 .+0$ ID =CDS:AT1G01110. 1; Parent=AT1G01110. 1
1 araport11 exon $5348453624 .+$. Parent=AT1G01110 1
1 araport11 CDS $\quad 5348453624 .+0$ ID=CDS:AT1G01110. 1 ; Parent=AT1G01110. 1
1 araport11 exon $\quad 5348453624 .+$. Parent=AT1G01110 2
1 araport11 CDS $\quad 5348453624 .+0$ ID=CDS:AT1G01110. 2 ; Parent=AT1G01110. 2

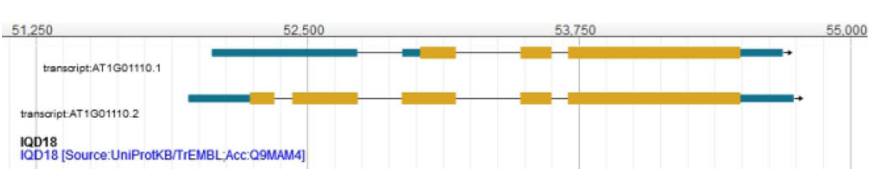

Fig. 1 The motivation for, outlines of, and action effects of GFF3sort. a An example of incorrectly sorted GFF3 data and how this is rendered in JBrowse. Blocks with the same start position are marked in blue-yellow stripes. The two lines (mRNA) marked in red were placed after their subfeatures (exon or UTR). Such an ordering leads to losing the first exon in JBrowse rendering results. See Additional file 2 for the full annotation lines. $\mathbf{b}$ Overview of GFF3sort. c An example of features sorted by GFF3sort and this is correctly rendered by JBrowse. In this example, the two lines (mRNA) marked in red are now placed before their sub-features, allowing JBrowse to render them correctly 
Here, we present GFF3sort, a novel tool to sort GFF3 files for tabix indexing. Compared with GNU sort and GenomeTools, GFF3sort produces sorting results that can be correctly rendered by JBrowse while still has comparable time and memory requirements. We anticipate that GFF3sort will be a useful tool to help with processing and visualizing genome annotation data.

\section{Implementation}

GFF3sort is a script written in Perl. It uses a hash table to store the input GFF3 annotation data (Fig. 1b). For each feature, the chromosome ID and the start position are stored in the primary and secondary key, respectively. Features with the same chromosome and start position are grouped in an array in the same order of their appearance in the original GFF3 data. After sorting the hash table by chromosome IDs and start positions, GFF3sort implemented two modes to sort features within the array: the default mode and the precise mode (Fig. 1b). In most situations, the original GFF3 annotations produced by genome annotation projects have already placed parent features before their children. Therefore, GFF3sort returns the feature lines in their original order, which is the default behavior. In some situations where orders in the input file has not yet placed parent features before child features, GFF3sort would replace them according to the parent-child topology using the sorting algorithm of directed acyclic graph [9], which is the most precise behavior but costs a little more computational time.

In order to test the performance of GFF3sort, the GFF3 annotation files of seven species, Saccharomyces cerevisiae (R64-1-1), Aspergillus nidulans (ASM1142v1), Chlamydomonas reinhardtii (INSDC v3.1), Drosophila melanogaster (BDGP6), Arabidopsis thaliana (Araport11), Rattus norvegicus (Rnor_6.0), and Homo sapiens (GRCh38), were downloaded from the ENSEMBL database [10]. All the tests were conducted on a SuperMicro ${ }^{\circ}$ server equipped with $80 \mathrm{Intel}^{\odot} \mathrm{Xeon}^{\oplus} \mathrm{CPUs}(2.40 \mathrm{GHz})$, 128 GB RAM, and running the CentOS 6.9 system. By default, CentOS 6.9 carries GNU sort v8.4, a relatively old version released in 2010. Therefore, we downloaded and installed a new version (v8.28) from the official repository of GNU Coreutils [11]. Both the old and the new version of GNU sort are used in benchmarking.

\section{Results and discussion}

GFF3sort takes a GFF3 file as its input data and returns a sorted GFF3 file as output. Several optional parameters are provided such as turning on the precise mode, sorting chromosomes in different ways and properly dealing with inline FASTA sequences. Features sorted by GFF3sort are correctly rendered by JBrowse (Fig. 1c and Additional file 2).
In addition to providing a sort order that correctly renders in JBrowse, GFF3sort has also other advantages over traditional tools. Compared with the GNU sort program, GFF3sort can properly deal with GFF3-specific lines or directives that are preceded by the '\#\#' symbol, such as the topmost GFF version line, the sequenceregion lines, and the embedded FASTA sequences. Compared with the GenomeTools, GFF3sort runs significantly faster (Additional file 1). In the default mode, GFF3sort saves $\sim 70 \%$ running time in our seven test datasets. The precise mode takes longer to run but is still faster than GenomeTools, especially for large annotation data such as human. While keeping a high running speed, the memory consumption is still acceptable (Additional file 1). For the largest annotation dataset (the GRCh38 annotation version of human) with a $\sim 400 \mathrm{MB}$ GFF3 file, the memory usage of GFF3sort is $\sim 758 \mathrm{MB}, \sim 40 \%$ less than GenomeTools.

\section{Conclusions}

In conclusion, GFF3sort is a novel tool to sort GFF3 files for tabix indexing and therefore can be used to visualize annotation data in JBrowse appropriately. It has a fast running speed compared with similar, existing tools. We anticipate that GFF3sort will be a useful tool to simplify data processing and visualization.

\section{Availability and requirements}

Project name: GFF3sort.

Project home page: https://github.com/billzt/gff3sort

Operating system(s): Linux.

Programming language: Perl.

Other requirements: No.

License: No restrictions for academic users.

Any restrictions to use by non-academics: license needed.

\section{Additional files}

Additional file 1: Benchmark data. This file displays: 1) the detailed running time of GFF3-to-JSON conversion and the bgzip-tabix process on our test datasets; 2) the detailed running time and 3) memory usage of GFF3sort, GNU sort (v8.4 and v8.28), and GenomeTools on our test datasets. (PDF $720 \mathrm{~kb}$ )

Additional file 2: The full GFF3 annotation lines used in Fig. 1a and c. It is the gene AT1G01110 extracted from the Arabidopsis thaliana (Araport11) annotation files. It includes three plain-text files: raw.gff3, GNUsort.gff3 (Fig. 1a), and GFF3sort.gff3 (Fig. 1c). (ZIP 2 kb)

Abbreviations

GFF3: General Feature Format, version 3; HTML5: HyperText Markup Language, version 5; JBrowse: JavaScript-based genome browser; JSON: JavaScript Object Notation

Acknowledgements

We thank Dr. Miklos Csuros and other anonymous reviewers for their helpful comments. 


\section{Funding}

This work is supported by grants from the National Natural Science

Foundation of China (Grant No. 31771850) and the Ministry of Agriculture of China (Grant No. 2016ZX08005004).

\section{Authors' contributions}

$S G, R Z$, and TZ initiated the idea of the tool and conceived the project. TZ designed the tool and analyzed the data. $\mathrm{CL}$ and $\mathrm{ZM}$ helped to test the tool. TZ wrote the paper. All authors read and approved the final manuscript.

Ethics approval and consent to participate

Not applicable.

\section{Consent for publication}

Not applicable.

\section{Competing interests}

The authors declare that they have no competing interests.

\section{Publisher's Note}

Springer Nature remains neutral with regard to jurisdictional claims in published maps and institutional affiliations.

Received: 29 June 2017 Accepted: 6 November 2017

Published online: 14 November 2017

\section{References}

1. Skinner ME, Uzilov AV, Stein LD, Mungall CJ, Holmes IH. JBrowse: a nextgeneration genome browser. Genome Res. 2009;19(9):1630-8.

2. Buels R, Yao E, Diesh CM, Hayes RD, Munoz-Torres M, Helt G, Goodstein DM, Elsik CG, Lewis SE, Stein L, et al. JBrowse: a dynamic web platform for genome visualization and analysis. Genome Biol. 2016;17(1):66.

3. JBrowse Configuration Guide [http://gmod.org/wiki/JBrowse_Configuration Guide] Accessed 26 May 2017.

4. JBrowse-1.12.3: Maintenance Release [http://jbrowse.org/jbrowse-1-12-3/ Accessed 26 May 2017.

5. Li H. Tabix: fast retrieval of sequence features from generic TAB-delimited files. Bioinformatics. 2011;27(5):718-9.

6. Gremme G, Steinbiss S, Kurtz S. GenomeTools: a comprehensive software library for efficient processing of structured genome annotations. IEEE/ACM Trans Comput Biol Bioinformatics. 2013;10(3):645-56.

7. JBrowse FAQ [http://gmod.org/wiki/JBrowse_FAQ] Accessed 26 May 2017.

8. Potential GFF3Tabix issues [https://github.com/GMOD/jbrowse/issues/780] Accessed 26 May 2017.

9. Sort::Topological - Topological Sort - metacpan.org [https://metacpan.org/ pod/Sort::Topological] Accessed 4 June 2017.

10. Aken BL, Achuthan P, Akanni W, Amode MR, Bernsdorff F, Bhai J, Billis K, Carvalho-Silva D, Cummins C, Clapham P, et al. Ensembl 2017. Nucleic Acids Res. 2017:45(D1):D635-42.

11. Coreutils - GNU core utilities [https:/www.gnu.org/software/coreutils/ coreutils.html] Accessed 15 Sept 2017.

\section{Submit your next manuscript to BioMed Central and we will help you at every step:}

- We accept pre-submission inquiries

- Our selector tool helps you to find the most relevant journal

- We provide round the clock customer support

- Convenient online submission

- Thorough peer review

- Inclusion in PubMed and all major indexing services

- Maximum visibility for your research

Submit your manuscript at www.biomedcentral.com/submit 